\title{
MiR-33 promotes myocardial fibrosis by inhibiting MMP16 and stimulating p38 MAPK signaling
}

\author{
Zhen Chen ${ }^{1,2,3}$, Hua-Sheng Ding ${ }^{1,2,3}$, Xin Guo ${ }^{1,2,3}$, Jing-Jing Shen ${ }^{1,2,3}$, Di Fan ${ }^{1,2,3}$, Yan \\ Huang $^{1,2,3}$ and Cong-Xin Huang ${ }^{1,2,3}$ \\ ${ }^{1}$ Department of Cardiology, Renmin Hospital of Wuhan University, Wuhan University, Wuhan 430060, PR China \\ ${ }^{2}$ Institute of Cardiovascular Diseases, Wuhan University, Wuhan 430060, PR China \\ ${ }^{3}$ Hubei Key Laboratory of Cardiology, Wuhan 430060, PR China \\ Correspondence to: Cong-Xin Huang, email: huangcongxin@vip. 163.com \\ Keywords: myocardial fibrosis; cardiac fibroblasts; miRNA-33; matrix metalloproteinase 16; p38 MAPK signaling pathway
}

Received: July 31, $2017 \quad$ Accepted: January 20, $2018 \quad$ Published: April 24, 2018

Copyright: Chen et al. This is an open-access article distributed under the terms of the Creative Commons Attribution License 3.0 (CC BY 3.0), which permits unrestricted use, distribution, and reproduction in any medium, provided the original author and source are credited.

\section{ABSTRACT}

Myocardial fibrosis occurs in the late stages of many cardiovascular diseases, and appears to be stimulated by various microRNAs (miRNAs). We previously found that miR-33 may stimulate cardiac remodeling. Here, we examined the involvement of miR-33 in myocardial fibrosis. Proximal left coronary descending artery occlusion was performed in rat, and antagomiR-33a was injected. Primary cardiac fibroblasts were cultured and transfected with miR-33a mimics and inhibitors. miR-33a levels were increased in the rat after surgery, and collagen deposition and heart fibrosis were observed in vivo. Inhibition of miR-33a suppressed fibroblast proliferation, reduced the mRNA and protein levels of collagen-related markers in vitro and in vivo, and rescued the histological damage in vivo. A dual-luciferase reporter system showed that matrix metalloproteinase 16 (MMP16) gene was the direct target of MiR-33a. These results suggest that miR-33 promoted myocardial fibrosis by inhibiting MMP16 and stimulating p38 mitogen-activated protein kinase (p38 MAPK) signaling pathway. MiR-33 may act as a novel therapeutic target for treating myocardial fibrosis.

\section{INTRODUCTION}

Myocardial fibrosis (MF) occurs in the late stages of many cardiovascular diseases, and it targets cardiac fibroblasts ( $\mathrm{CFs}$ ). Under normal conditions, $\mathrm{CFs}$ represent at least $60 \%$ of the total cells in the heart. Within a damaged heart, CFs are rapidly activated by microenvironment signals to remove dead cardiomyocytes, generate collagenenriched ECM, and repair the damaged areas [1]. In this process, cardiomyocytes showed negligible regenerative abilities, while CFs invaded and destroyed the architecture of the heart. Because CF activation promotes MF, CFs are a prospective therapeutic target to diminish MF [2].

MicroRNA (miR) is small non-coding RNA that inhibits gene expression at a post-transcriptional level by binding to the complementary sequences in the $3^{\prime}$ untranslated regions (3-UTRs) of their target genes [2, 3]. MicroRNAs can have anti-fibrotic functions, pro-fibrotic functions, or serve as MF biomarkers [2, 4]. MiR-33a, a highly conserved microRNA ubiquitously expressed in CFs [5], is a potential therapeutic target for cardiovascular diseases because of its correlation with high-density lipoprotein cholesterol (HDL-C) [6]. Anti-miR33 therapy inhibits mitochondrial respiration and ATP production, which in conjunction with increased ABCA1 expression, works to promote macrophage cholesterol efflux and reduce atherosclerosis [5]. We assessed miR-33's function in heart fibrosis.

\section{RESULTS}

MiR-33a was increased in vitro and in vivo

The Masson's trichrome staining and Sirius red staining of heart tissue sections both revealed typical collagen deposition at 28 days post-surgery (Figure 1A, 
$1 \mathrm{~B}, 1 \mathrm{D}, 1 \mathrm{E}, 1 \mathrm{G}$, and $1 \mathrm{H})$. We also found that CTGF, Col1A1, and Col3A1 levels increased in heart tissues according to qPCR (Figure 1J) and Western blot (Figure 1L). The same techniques revealed that Ang-II exposure in CFs resulted in more CTGF, Col1A1, and Col1A3 than in non-treated cells (Figure 1K, 1M). In addition, miR-33a levels increased in the heart tissues of MI (Figure 1J) and Ang-II induced CFs (Figure 1K).

\section{Inhibition of miR-33a improved echocardiographic function in vivo}

We evaluated miR-33a inhibition on LV remodeling and function via echocardiography (Figure 2A-2D). After permanent LAD occlusion, antagomiR-33a injection increased LVEF and LVFS levels, but mismatch antagomiR-33a (M-antagomiR-33a) injection did not (Figure 2E, 2F).

\section{Inhibition of miR-33a suppressed fibrogenesis in vitro and in vivo}

To investigate if miR-33a inhibition inhibited fibrogenesis, we injected antagomiR-33a to animals through the tail vein. AntagomiR-33a inhibition reduced miR-33a levels in heart tissues (Figure 3A), rescued the histological damage (Figure 1C, 1F, 1I), and reduced the mRNA and protein levels of CTGF, Col1A1 and Col3A1 (Figure 3B-3E). However, mismatch antagomiR33a (M-antagomiR-33a) failed to show these effects (Figure 3B-3E).

MiR-33a inhibition in CFs by miR-33a inhibitor reduced proliferation rate (Figure $4 \mathrm{~A}$ ) and lowered mRNA levels of miR-33a, CTGF, Col1A1, and Col3A1 (Figure 4B-4E) compared with non-treated cells by qPCR. Inhibition of miR-33a in CFs also reduced protein levels of CTGF, Col1A1, and Col3A1 by Western blot (Figure 4F). Overexpression of miR-33a in CFs by miR-33a mimics increased RNA and protein levels of CTGF, Col1A1, and Col3A1 by qPCR and Western blot (Figure 4C-4F).

\section{MiR-33a mediated fibrogenesis via the direct target gene, MMP16}

To confirm potential miR-33a target genes, we used the online prediction programs TargetScan (www. targetscan.org) and miRBase (www.microrna.org). Among all these potential genes, MMP16 was considered to promote the fibrosis process [7, 8]. MMP16 levels in heart tissues reduced after surgery (Figure 5A). To verify whether miR-33a directly binds to the 3'-UTR of rat MMP16 mRNA in the post-transcriptional level, we cloned the wild-type and mutated 3'- UTR sequence of MMP16 into a dual-luciferase reporter vector. Wildtype 3'-UTR of MMP16 (MMP16-3'-UTR) reduced the luciferase activity in the presence of miR-33a, whereas
miR-33a did not affect the luciferase activity of the mutated 3'-UTR of MMP16 (MMP16-3'-UTR-mut) (Figure 5B). MMP16 overexpression inhibited miR-33a mimics-induced increases of CTGF, Col1A1, and Col3A1 in CFs (Figure 5C-5E). However, similar effects were not observed using empty control (data not shown).

\section{MiR-33a controlled fibrogenesis through $\mathrm{p38}$ MAPK but not TGF-ק/Smad pathway}

Myocardial fibrosis can be promoted by the p38 MAPK [9] and TGF- $\beta /$ Smad signaling pathways [10]. We examined whether miR-33a used either of those signaling pathways to stimulate myocardial fibrogenesis. We found that surgery in rat increased phospho-p38 MAPK levels at 28 days post-surgery, and antagomiR-33a injection inhibited this pathway (Figure 6A). While Smad3 and TGF- $\beta 1$ protein levels in the TGF- $\beta /$ Smad signaling pathway were also increased in the animals at 28 days post-surgery, they were not reduced by antagomiR33a injection (Figure 6B). Smad3 phosphorylation (Ser423/425) was also not suppressed by antagomiR33a injection (Figure 6B). In contrast, Smad4 protein decreased in the animals at 28 days post-surgery, and were not increased by antagomiR-33a injection (Figure 6B).

\section{DISCUSSION}

A series of miRNAs are strongly associated with the pathogenesis of myocardial fibrosis, including miR-33a [11-17]. MiR-33a and miR-33b are conserved miRNA located in intron 16 of SREBP-2 and SREBP-1, respectively [18]. They are the most abundant miRNAs in lipoprotein particles [19]. Cardiac fibrosis can be ameliorated in miR33 knockout hearts, and cardiac fibroblasts (CFs) are mainly responsible for miR-33 expression in the heart [5]. Longterm therapeutic silencing of miR-33 increases circulating triglyceride levels and hepatic lipid accumulation in mice [20], and genetic permanent miR-33 inhibition may cause cardiac dysfunction [5]. Considering these harmful side effects, we adopted the tentative silencing of miR-33 approach by antagomiR-33a. No prominent side effects were observed. In the present study, miR-33a was increased in rat hearts after myocardial infarction. Additionally, miR33a inhibition suppressed cardiac fibrosis in vivo, as well as collagen formation and fibrogenesis in vitro, by targeting MMP16.

We used online prediction programs to predict all target genes of miR-33, and MMP16 was screened for further research because MMPs are a group of proteolytic enzymes that are responsible for the maintenance of the ECM [21]. MMP16 expression is decreased in dupuytren's disease patients, which is a typical fibrosis disease [8]. Decreased MMP16 levels have also been verified in Ang II-induced miRNAs in cardiac fibroblasts [22]. MMP16 protein activates MMP2 protein which in turn degrades 

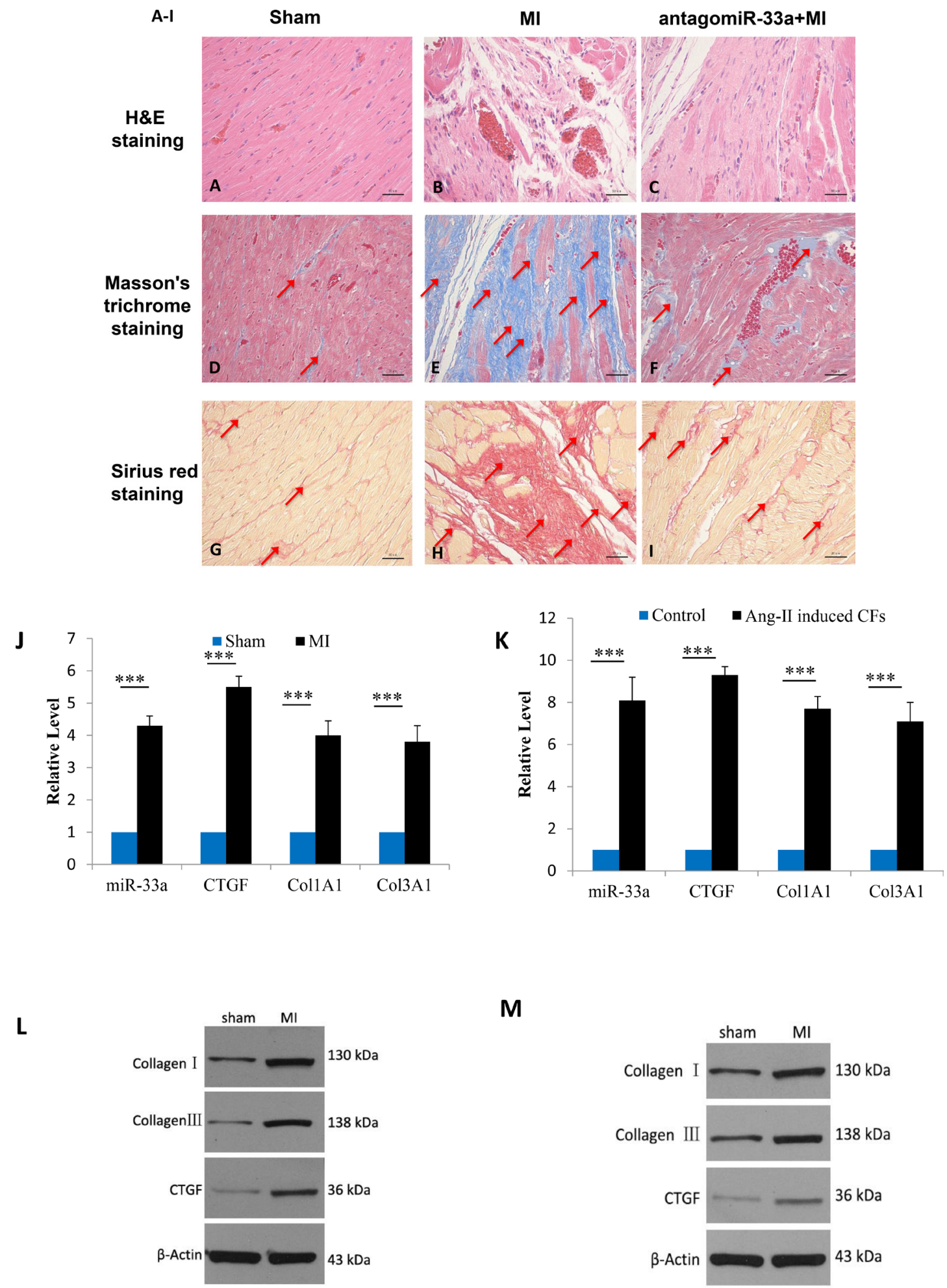

Figure 1: MiR-33a was increased in vitro and in vivo; myocardial fibrosis observed in vivo. Representative micrographs of H\&E-stained (A-C, top row), Masson's trichrome-stained (D-F, middle row) and Sirius red-stained (G-I, bottom row) heart tissue sections from sham operated- (left column), myocardial infarction (MI) (middle column), and antagomiR-33a injected- (right column) animals. (J) The mRNA levels of miR-33a, CTGF, Col1A1, and Col3A1 in heart tissues were examined by qPCR between MI and sham operated rats. (K) The mRNA levels of miR-33a, CTGF, Col1A1, and Col3A1 in CFs were examined by qPCR between Ang-II-induced CFs and normal CFs. (L) The protein levels of CTGF, Col1A1, and Col3A1 in heart tissues were examined by Western blot between MI and sham operated rats. (M) The protein levels of CTGF, Col1 A1, and Col3A1 in CFs were examined by Western blot between Ang-IIinduced CFs and normal CFs. Arrows indicate collagen deposition in (D-I). Data are expressed as mean $\pm \operatorname{SD}(n=3){ }^{*} p<0.05,{ }^{* *} p<0.01$, ${ }^{* * *} p<0.001$, no significance is indicated as "NS". Scale bars: $50 \mu \mathrm{m}$. 

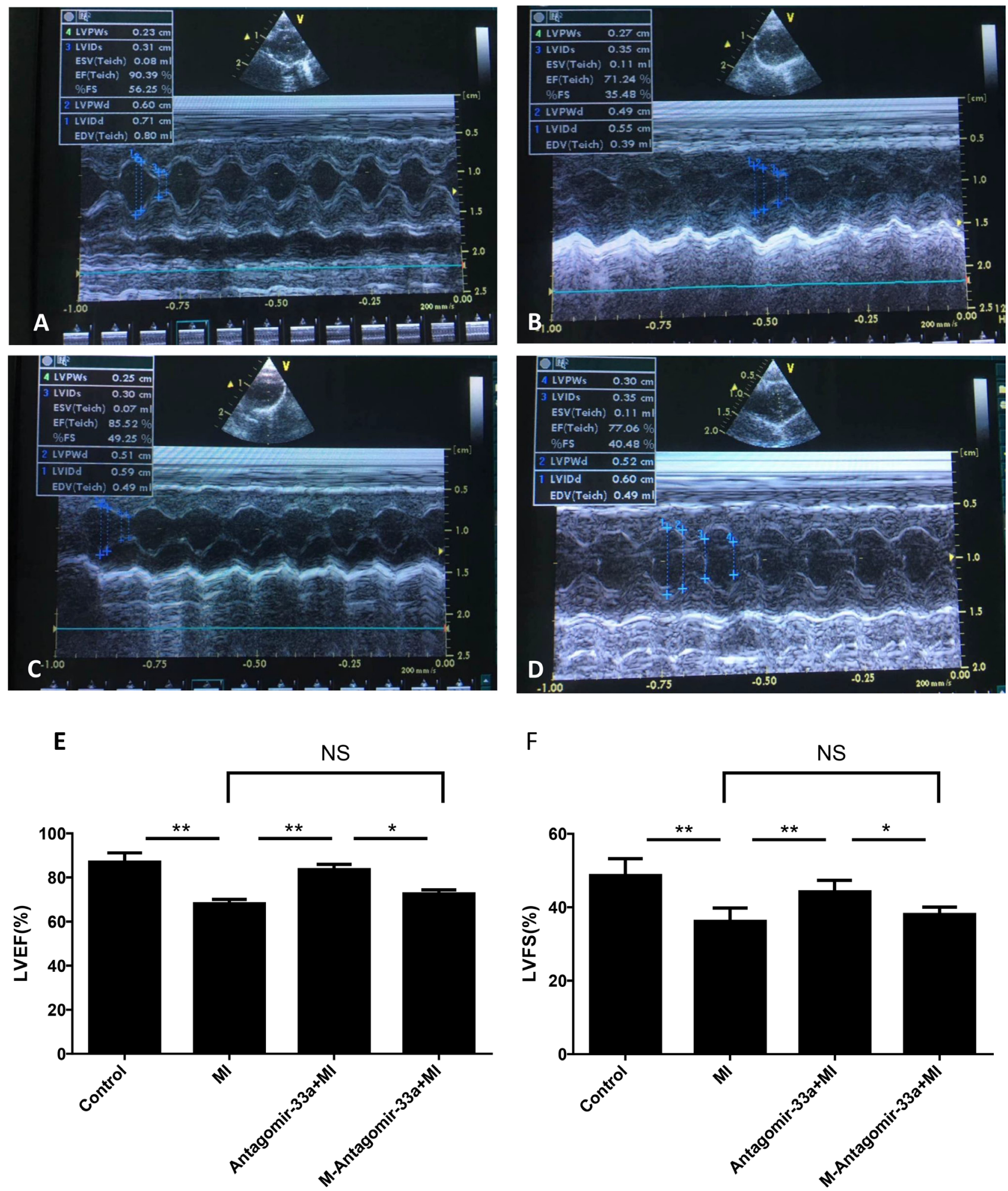

Figure 2: Inhibition of miR-33a improved echocardiographic function in vivo. Echocardiography was performed at the end of the study $(n=8)$. Representative M-mode images of sham operated- (A), myocardial infarction (MI)- (B), antagomiR-33a injected- (C), and mismatch antagomiR-33a (M-antagomiR-33a) (D) animals. LVEF (E) and LVFS (F) were obtained. Data are expressed as mean \pm SD $(n=3) .{ }^{*} p<0.05,{ }^{* *} p<0.01,{ }^{* * *} p<0.001$, no significance is indicated as "NS". 

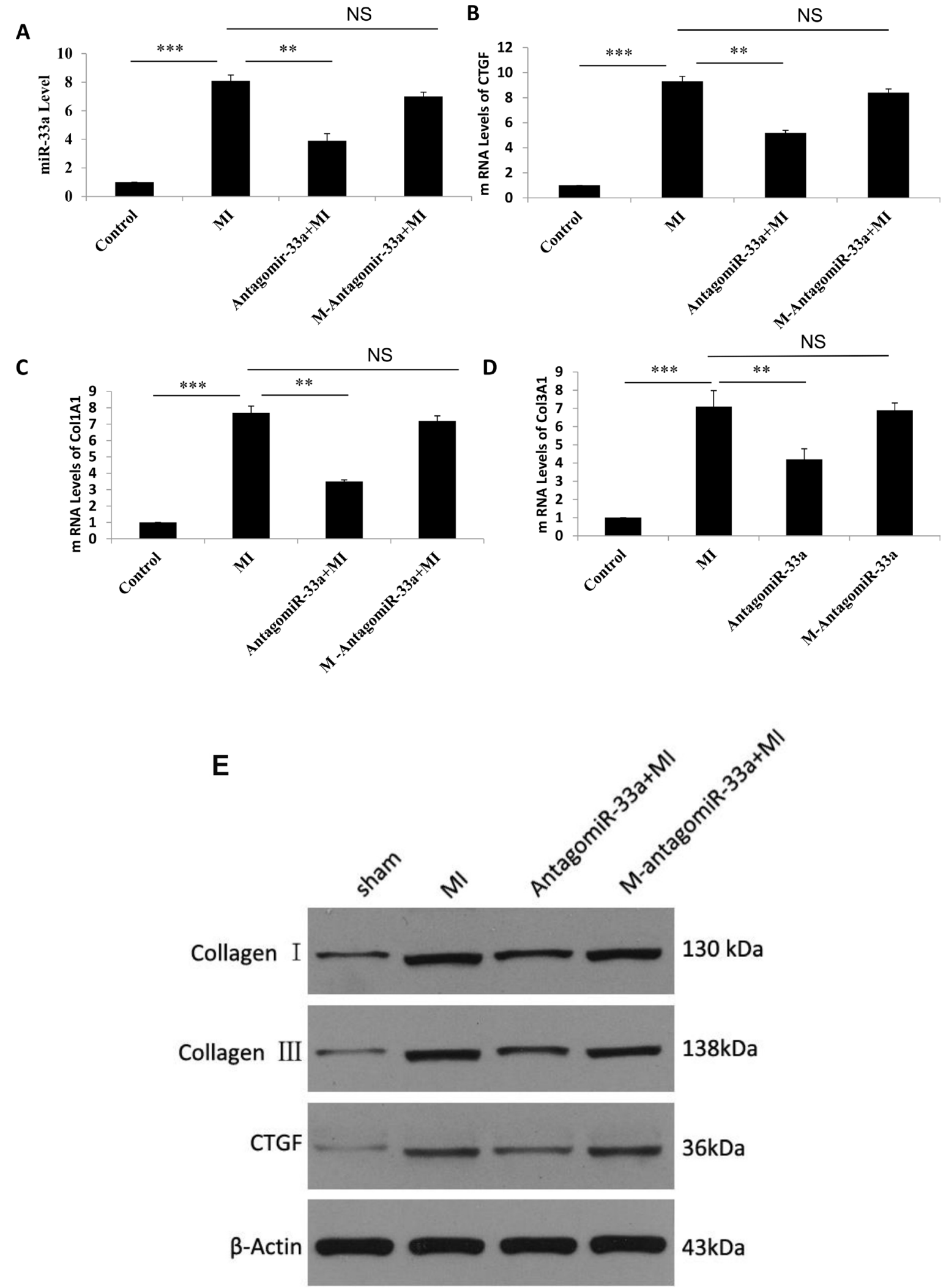

Figure 3: Inhibition of miR-33a suppressed fibrogenesis in vivo. The mRNA levels of miR-33a (A), CTGF (B), Col1A1 (C), and Col3A1 (D) in heart tissues were examined by qPCR among sham rats (Control), MI rats (MI), antagomiR-33a injected MI rats (antagomiR-33a+MI), and mismatch antagomiR-33a injected MI rats (M-antagomiR-33a+MI). (E) The protein levels of CTGF, Col1A1, and Col3A1 in heart tissues were examined by Western blot among sham, MI, antagomiR-33a+MI, and M-antagomiR-33a+MI. Data are expressed as mean $\pm \operatorname{SD}(n=3) . " p<0.05,{ }^{* *} p<0.01,{ }^{* * *} p<0.001$, no significance is indicated as "NS". 

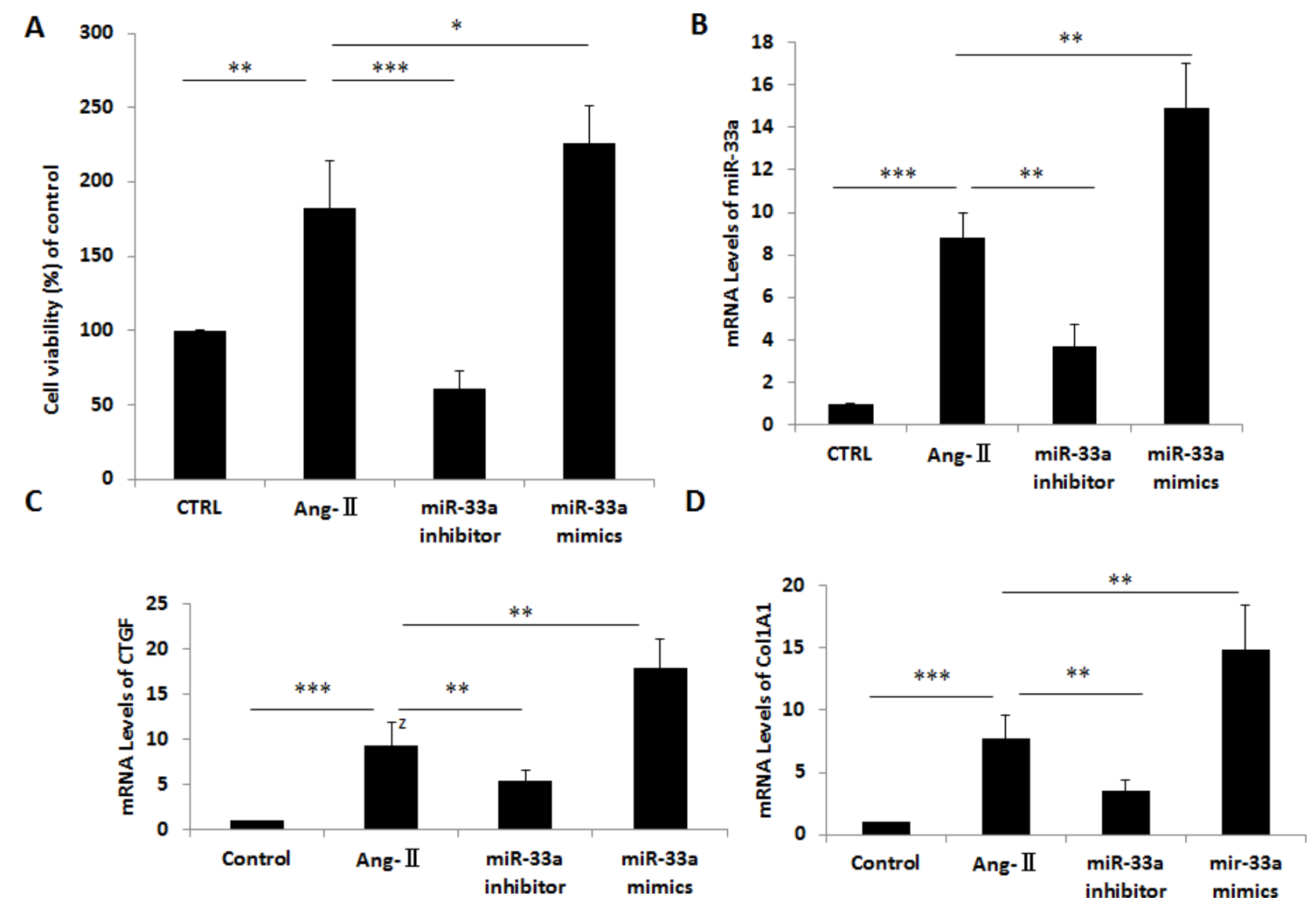

E

$$
\mathbf{F}
$$
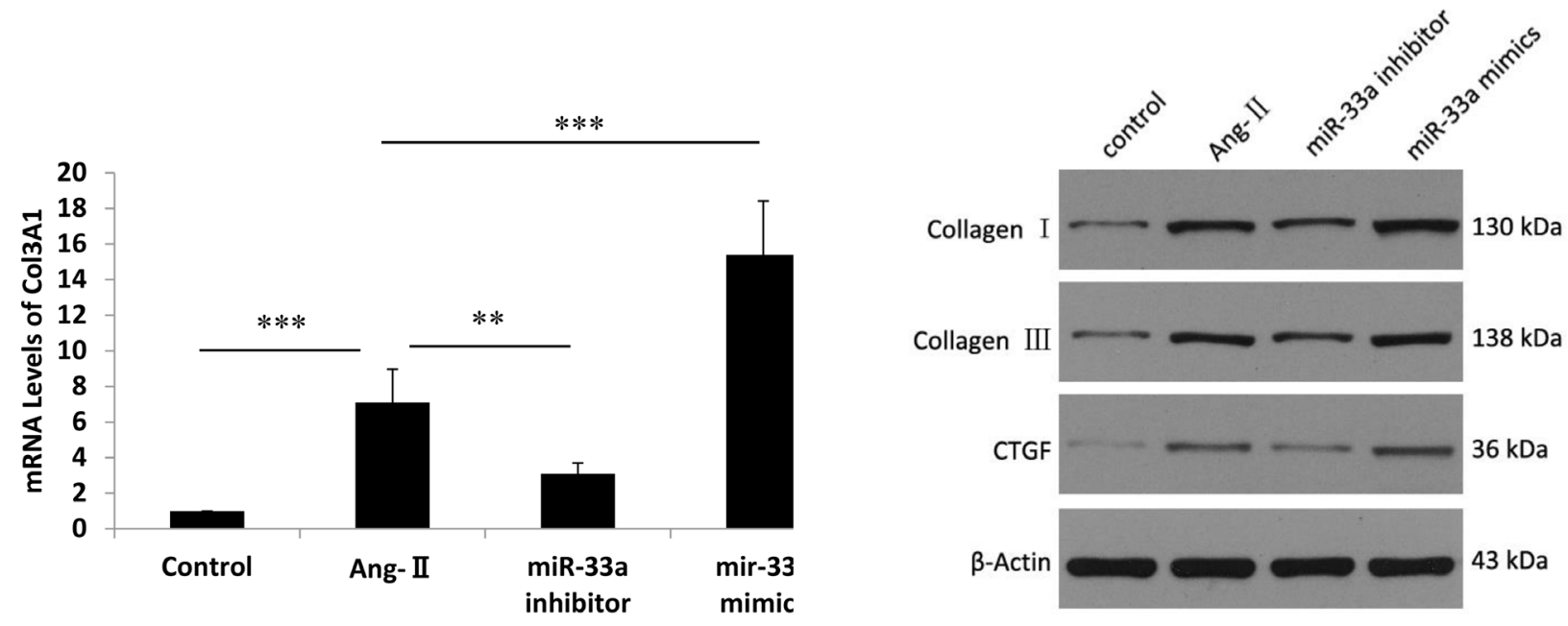

Figure 4: Inhibition of miR-33a suppressed fibrogenesis in vitro. (A) Cell viability was analyzed by CCK8 in control cells (CTRL), Ang-II-induced CFs (Ang-II), miR-33a inhibitor treated CFs (miR-33a inhibitor), and miR-33a mimics treated CFs (miR-33a mimics). (B-E) The mRNA levels of miR-33a (B), CTGF (C), Col1A1 (D), and Col3A1 (E) were examined by qPCR in control cells (CTRL), Ang-II-induced CFs (Ang-II), miR-33a inhibitor treated CFs (miR-33a inhibitor), and miR-33a mimics treated CFs (miR-33a mimics). (F) The protein levels of CTGF, Col1A1, and Col3A1 were examined by Western blot in control cells (CTRL), Ang-II induced CFs (Ang-II), miR-33a inhibitor treated CFs (miR-33a inhibitor), and miR-33a mimics treated CFs (miR-33a mimics). Data are expressed as mean $\pm \mathrm{SD}(n=3) .{ }^{*} p<0.05,{ }^{* *} p<0.01,{ }^{* * *} p<0.001$, no significance is indicated as "NS". 

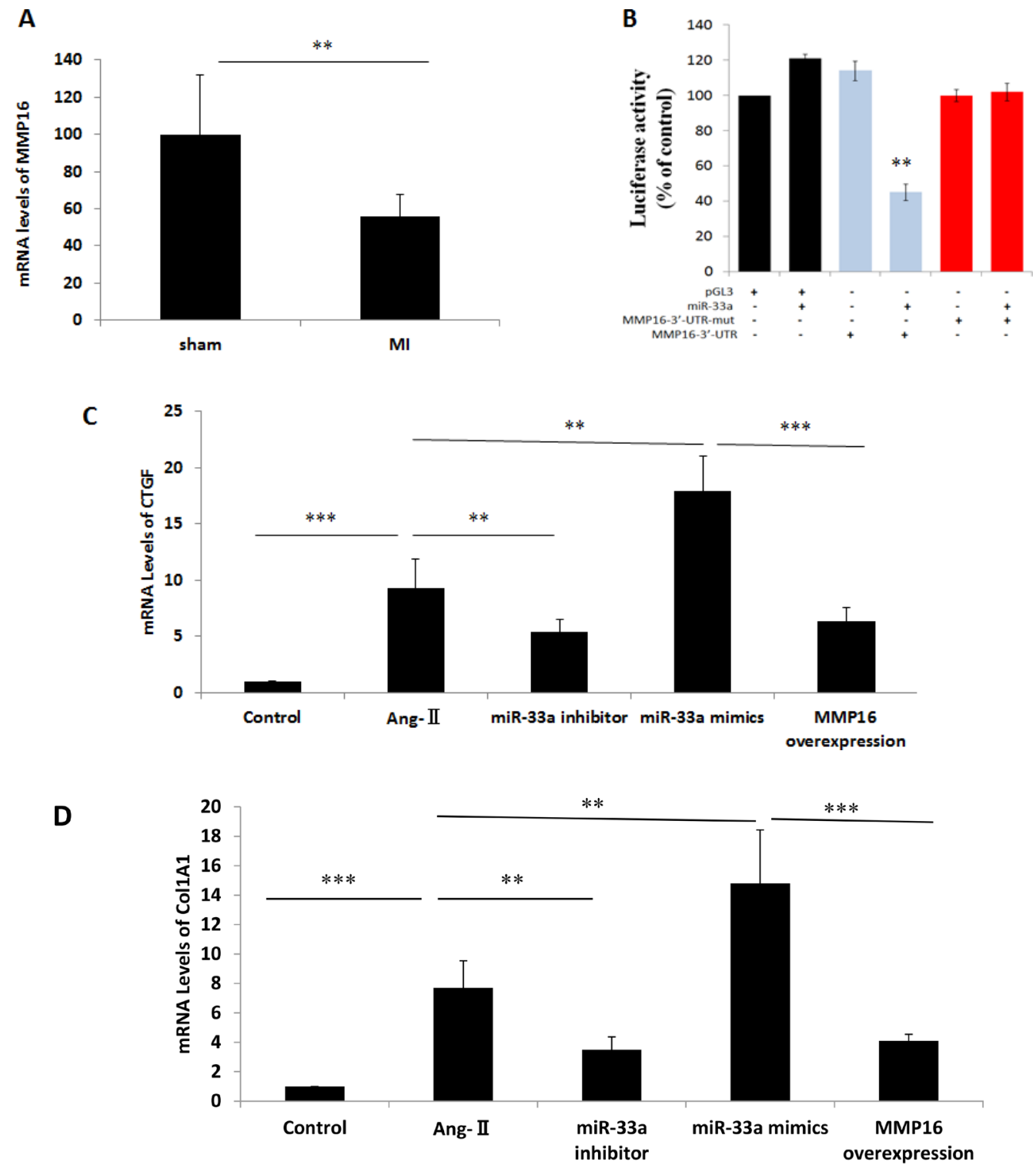

E

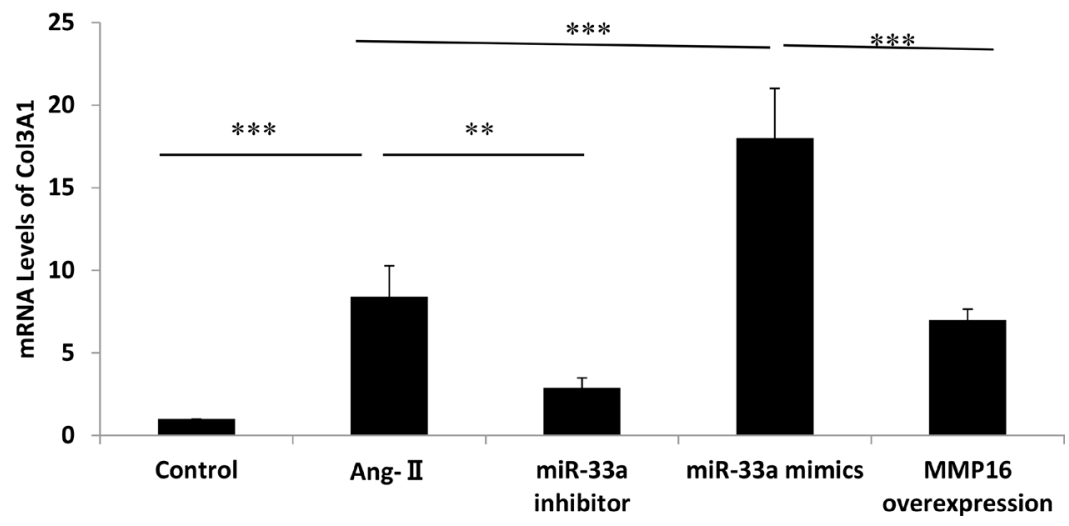

Figure 5: MiR-33a promoted fibrogenesis via its direct target gene, MMP16. (A) The mRNA levels of MMP16 in heart tissues were examined by qPCR between sham rats, and MI rats (MI). (B) Validation of MMP16 as a miR-33 target gene by luciferase-activity assay. Introduction of mutations in the 3'-UTR of MMP16 blocked inhibition of MMP16 translation. (C-E) The mRNA levels of CTGF (C), Col1A1 (D), and Col3A1 (E) were examined by qPCR in control cells (CTRL), Ang-II-induced CFs (Ang-II), miR-33a inhibitor treated CFs (miR-33a inhibitor), and MMP16 over-expressed CFs (MMP16 overexpression). Data are expressed as mean \pm SD $(n=3)$. " $p<0.05,{ }^{* *} p<0.01,{ }^{* * *} p<0.001$, no significance is indicated as "NS". 
type III collagen [8]. We demonstrated that MMP16 levels decreased in the heart tissues, and MMP16 overexpression inhibited miR-33a mimics-induced increases of CTGF, Col1A1, and Col3A1 in CFs. Our dual-luciferase reporter assay confirmed that MMP16 was a direct target gene of miR-33a.

TGF- $\beta /$ Smad signaling stimulates myocardial fibrogenesis [10]. Western blot analysis revealed that miR33a/MMP16 did not promote fibrogenesis through TGF- $\beta$ / Smad signaling. Previous studies demonstrated that TGF- $\beta$ /Smad signaling interacted with MAPK signaling in fibrosis disease [23]. We then examined whether miR33a controlled fibrogenesis through MAPK signaling by western blot, and found that phosphorylated p38 was reduced in the antagomiR-33a injection-treated heart tissues more than the un-treated heart tissues.

We performed a comprehensive study of miR-33 expression in a rat cardiac fibrosis model in vivo, Ang-IItreated primary cardiac fibroblasts in vitro, and miR-33 target genes. We found miR-33a was increased in the rat after surgery, and collagen deposition and heart fibrosis were observed in vivo simultaneously. We also found that miR-33a inhibition inhibited CF proliferation rate, reduced the mRNA and protein levels of collagen-related markers in vitro and in vivo, and rescued the histological damage in vivo. We obtained the opposite results when miR-33a was over-expressed in cultured CFs by miR-33a mimics. Furthermore, we found that MMP16 gene was the direct target for miR-33a in CFs, and that miR-33a promoted cardiac fibrosis via the p38 MAPK signaling pathway.

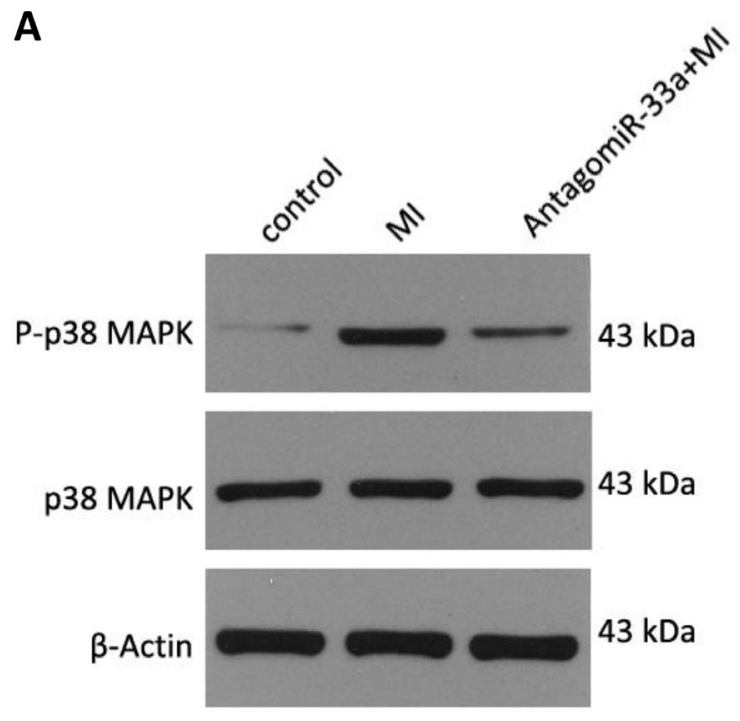

Our results indicate miR-33a could be a novel therapeutic target for myocardial fibrosis.

\section{MATERIALS AND METHODS}

\section{Animal welfare and ethical approval}

Eight-week-old Sprague-Dawley (SD) rats were housed according to United States National Institutes of Health (NIH) guidelines and the local committee for the care and use of laboratory animals. The rats were housed three rats per cage prior to surgery, and one rat per cage after surgery. All surgical procedures and animal experiments were approved by the welfare committee for animal use and care, and performed according to the Guide for the Care and Use of Laboratory Animals published by the NIH. Every effort was made to minimize the number of animals used, as well as their suffering.

\section{Animal model of myocardial fibrosis and miR- 33a treatment}

SD rats were anesthetized with $3 \%$ sodium pent barbiturate $(50 \mathrm{mg} / \mathrm{kg})$, intubated with a polyethylene catheter, and room air was continuously provided by a rodent ventilator. The chest was opened by left thoracotomy between the third and the fourth intercostal space, and the pericardium was opened and removed. The proximal left coronary descending artery (LAD) was permanently encircled and ligated with an intramural

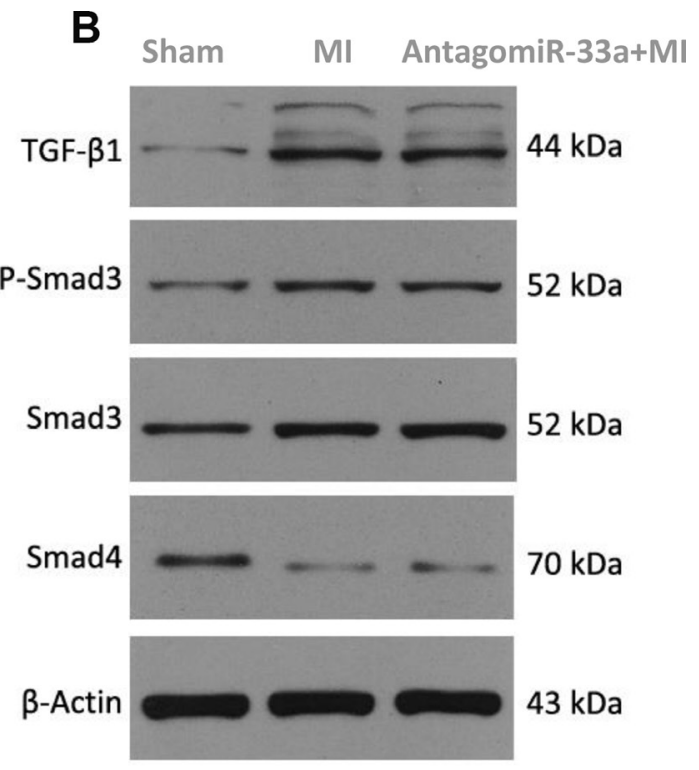

Figure 6: MiR-33a stimulates fibrogenesis through p38 MAPK but not TGF- $\boldsymbol{\beta}$ /Smad pathway. (A) The protein levels of phospho-p38 MAPK, total p38 MAPK in heart tissues were examined by Western blot among sham rats (Control), MI rats (MI), antagomiR33a injected MI rats (antagomiR-33a+MI). (B) The protein levels of TGF- $\beta 1$, phospho-Smad3, Smad3, Smad4 in heart tissues were examined by Western blot among sham rats (Control), MI rats (MI), antagomiR-33a injected MI rats (antagomiR-33a+MI). Data are expressed as mean $\pm \mathrm{SD}(n=3) .{ }^{*} p<0.05,{ }^{* *} p<0.01,{ }^{* * *} p<0.001$, no significance is indicated as "NS". 
stitch, then the chest was closed. High mortality occurred during induction procedures, and rats that died within $24 \mathrm{~h}$ after surgery were excluded for further analysis. Shamoperated rats were subjected to the same procedure, but without ligation.

The cholesterol-conjugated miR-33a antisense (antagomiR-33a) and mismatch antagomiR-33a (M-antagomiR-33a) were purchased from RiboBio Co., Ltd. (Guangzhou, China) [7, 8, 11, 24]. The antagomiR33a and M-antagomiR-33a were injected through the tail vein at $40 \mathrm{mg} / \mathrm{kg}$ for seven consecutive days after surgery [7, 24]. These constructs were chemically modified and conjugated with cholesterol moiety for in vivo applications with long-lasting stability and enhanced target specificity and affinity [11].

\section{Echocardiography}

Echocardiographic analyses were performed 28 days after operation. Rats were anesthetized with 50 $\mathrm{mg} / \mathrm{kg}$ ketamine and $10 \mathrm{mg} / \mathrm{kg}$ xylazine. The chest was shaved, and the rats were placed supine. Echocardiograms were performed with a commercially available echocardiography system equipped with 7.5- MHz phasedarray transducer (Hewlett-Packard). The transducer was positioned on the left anterior side of the chest after the precordium was shaved. We evaluated the left ventricle (LV) dimensions in parasternal short-axis view during systole or diastole. We measured left ventricular fractional shortening (LVFS), left ventricular ejection fraction (LVEF) from the LV M-mode tracing with a sweep speed of $50 \mathrm{~mm} / \mathrm{s}$ at the mid-papillary muscle level.

\section{Sample collection and histological analysis}

The rats were sacrificed 28 days post-surgery. Some hearts were fixed with $4 \%$ paraformaldehyde for histological and immunohistochemical analysis, while some hearts were snap frozen with dry ice powder and stored in liquid nitrogen tank until use for qPCR and Western blot analysis. The hearts were dehydrated in ethanol, embedded in paraffin, sectioned at $4 \mu \mathrm{m}$ thickness, and stained with hemotoxylin/eosin (H\&E), Masson's trichrome, and Sirius red.

\section{Primary cardiac fibroblasts culture}

Primary CFs were isolated and purified according to previously described methods [11]. Newly dissected hearts were collected from 1 to 3-day-old SD rats, and immediately rinsed in cold PBS several times until the blood was cleared. The hearts were minced, and then digested with $0.25 \%$ trypsin and $0.02 \%$ EDTA (Sigma, USA) at $37^{\circ} \mathrm{C}$ for $1 \mathrm{~h}$. The digestion was stopped by adding Dulbecco's Modified Eagle's Medium (DMEM) (Hyclone, Logan, UT, USA) medium with 10\% fetal bovine serum (FBS) (Hyclone), $100 \mathrm{U} / \mathrm{ml}$ penicillin, and $100 \mathrm{ug} / \mathrm{ml}$ streptomycin. The pellets were gently re-suspended with $10 \% \mathrm{FBS} / \mathrm{DMEM}$, and then cultured in plates for $2 \mathrm{~h}$ at $37^{\circ} \mathrm{C}$ in a $\mathrm{CO}_{2}$ incubator. The nonadherent cardiomyocytes were gently changed off with $10 \% \mathrm{FBS} / \mathrm{DMEM}$, and the attached cells were cultured. At $70-80 \%$ confluency, cells were passaged, and cells at passage 2-6 were used for in vitro studies.

\section{CFs transfection and ang-II treatment}

CFs at $70-80 \%$ confluency were replaced and incubated with serum-free medium for $24 \mathrm{~h}$ before transfection. The cells were then transfected with miR33a mimic, miR-33a inhibitor (anti-miR-33a), or the negative control (NC) (100 nM each final concentration, Invitrogen, USA) by Lipofectamin 2000 according to the manufacturer's protocols (Invitrogen, Carlsbad, CA). After transfection, CFs were washed twice with PBS, replaced with fresh $10 \%$ FBS/DMEM medium, and treated with $10^{-7} \mathrm{~mol} / \mathrm{L}$ Ang-II for $24 \mathrm{~h}$ [24].

\section{Overexpression of MMP16 in CFs}

CFs were seeded at $1 \times 10^{5} /$ well in 6-well plates, then transfected with MMP16 overexpression plasmid (pEGFPN1-MMP16) or empty plasmid (pEGFP-N1) (GenePharma, Shanghai, China) by Lipofectamin 2000 (Invitrogen, Carlsbad, CA) according to the manufacturer's instructions.

\section{Cell viability assay}

CF cell viability was measured by $\mathrm{CCK} 8$ assay (cell counting kit-8, Dojindo Molecular Technologies, Tokyo, Japan) according to the manufacturer's instructions. CFs were seeded at $6 \times 10^{3}$ cells per well in 96-well plates and treated as described above. CCK-8 solution (10 $\mu \mathrm{l} /$ well) was added to the wells, and the plate was incubated for $2 \mathrm{~h}$ at $37^{\circ} \mathrm{C}$. The absorbance of each well was determined at $450 \mathrm{~nm}$ using a microplate reader, and the growth curve was calculated.

\section{Reverse transcription real-time polymerase chain reaction ( $R T-q P C R)$}

CFs were treated and collected as described above. Total RNA was extracted from CFs and heart tissues by Trizol reagent (TaKaRa, Tokyo, Japan). First strand complementary DNA (cDNA) was synthesized using the Reverse Transcription Kit (TaKaRa, Tokyo, Japan) according to manufacturer's instructions. For quantitative PCR (qPCR), PCR amplifications were quantified using the SYBR Green PCR Master Mix (Applied Biosystems) and normalized to GAPDH gene expression. MiR-33a quantification was performed with a stem-loop real-time PCR miRNA kit (Ribobio, Guangzhou, China).

The sequences of the q-PCR primers are described below: miR-33a (forward: 5'-GATCCTCAGTGCATTGT 
AGTTGC-3'; reverse: 5'-CTCTGTCTCT CGTCTTGTTG GTAT-3'), Col1A1 (forward: 5'-TCCTGACGCATGGCC AAGAA-3'; reverse: 5'-CATAGCACGCCATCGC ACAC-3'), Col3A1 (forward: 5'-TGGACAGATGCTGGT GCTGAG -3'; reverse: 5'-GAAGGCCAGCTGTACATC AAGGA-3'), CTGF (forward: 5'-GCAGCTAGAGAAGCA GAGC-3'; reverse: 5'-GGTGCAGCCAGAAAGCTC-3'), MMP16 (forward: 5'-AATCTCCTCAGGGAGCATTT GTA-3'; reverse: 5'-TCCAGGTTCTACC TTGAGTAT CTG-3'), U6 (forward: 5'-ATTGGAACGATACAGAG AAGATT-3'; reverse: 5'-GGAACGCTTCACGAATTT G-3'), and GAPDH (forward: 5'-GAC ATG CCG CCT GGA GAAAC-3'; reverse: 5'-AGC CCA GGA TGC CCT TTA GT-3').

\section{Luciferase assays}

The wild-type 3'-UTR of rat MMP16 containing the putative binding site for miR-33a was amplified by PCR. The mutated 3'-UTR of rat MMP16 was generated using a QuikChange II XL site-directed mutagenesis kit (Stratagene, San Diego, CA, USA), and the constructs were verified by sequencing. Wild-type and mutated 3'UTR regions were then sub-cloned into the pGL3 vector immediately downstream of the luciferase gene stop codon (Promega, USA). HEK293 cells were co-transfected with $100 \mathrm{ng}$ of the plasmid constructs, MMP16-3'UTR, or MMP16-3'UTR-mut, and infected with plasmids expressing miR-33a or pGL3. After 48 hours, luciferase activities were measured with a dual-luciferase reporter assay kit (Promega) according to the manufacturer's instructions.

\section{Western blot}

Proteins were extracted with RIPA lysis buffer (Beyotime, Jiangsu, China) from homogenized frozen heart tissues, as well as cells. Protein concentrations were measured by Bradford assay. Equal protein amounts were loaded into SDS-PAGE gels and separated by electrophoresis. Primary antibodies were used to detect CTGF (1:1000, Sigma-Aldrich, USA), Collagen I (1:5000, Sigma-Aldrich, USA), Collagen III (1:5000, SigmaAldrich, USA), and actin (1:10000, Sigma-Aldrich, USA). Western blot bands were scanned and quantified by Image J software.

\section{Statistical analysis}

All experiments were conducted at least in triplicate, and representative data are expressed as the mean $\pm \mathrm{SD}$. The comparisons were evaluated by one-way analysis of variance and for significant relationships, post-hoc multiple comparisons between means were evaluated with the Turkey test. All statistical analyses were performed using SPSS statistics software, and $p<0.05$ was considered significant.

\section{Author contributions}

Z.C. conceived the study, performed the experiments, analyzed the data, and drafted the manuscript. H.D., X.G., J.C., Y.H., and D.F. performed the experiments, acquired data, and analyzed the data. C.H. designed the overall study, analyzed the data, and revised the manuscript. All authors reviewed the manuscript.

\section{ACKNOWLEDGMENTS}

We thank technicians in the laboratory for excellent support for animal surgery.

\section{CONFLICTS OF INTEREST}

The authors declare no conflicts of interest.

\section{FUNDING}

This work was supported by the National Key Basic Research Development Program of China (The “973” Program; No. 2012CB518604), the Fundamental Research Funds for the Central Universities of China (No. 2042015kf0229).

\section{REFERENCES}

1. Souders CA, Bowers SL, Baudino TA. Cardiac fibroblast: the renaissance cell. Circ Res. 2009; 105:1164-1176. https://doi.org/10.1161/CIRCRESAHA.109.209809.

2. Deng $\mathrm{P}$, Chen L, Liu Z, Ye P, Wang S, Wu J, Yao Y, Sun Y, Huang X, Ren L, Zhang A, Wang K, Wu C, et al. MicroRNA-150 Inhibits the Activation of Cardiac Fibroblasts by Regulating c-Myb. Cell Physiol Biochem. 2016; 38:2103-2122. https://doi.org/10.1159/000445568.

3. Du Y, Liu XH, Zhu HC, Wang L, Ning JZ, Xiao CC. MiR543 Promotes Proliferation and Epithelial-Mesenchymal Transition in Prostate Cancer via Targeting RKIP. Cell Physiol Biochem. 2017; 41:1135-1146. https://doi. org/10.1159/000464120.

4. Tijsen AJ, Pinto YM, Creemers EE. Circulating microRNAs as diagnostic biomarkers for cardiovascular diseases. Am J Physiol Heart Circ Physiol. 2012; 303:H1085-1095. https://doi.org/10.1152/ajpheart.00191.2012.

5. Nishiga M, Horie T, Kuwabara Y, Nagao K, Baba O, Nakao T, Nishino T, Hakuno D, Nakashima Y, Nishi H, Nakazeki F, Ide Y, Koyama S, et al. MicroRNA-33 Controls Adaptive Fibrotic Response in the Remodeling Heart by Preserving Lipid Raft Cholesterol. Circ Res. 2017; 120:835847. https://doi.org/10.1161/CIRCRESAHA.116.309528.

6. Alrob OA, Khatib S, Naser SA. MicroRNAs 33, 122, and 208: a potential novel targets in the treatment of obesity, diabetes, and heart-related diseases. J Physiol Biochem. 2017; 73:307-314. https://doi.org/10.1007/s13105-016-0543-z. 
7. Yu HD, Xia S, Zha CQ, Deng SB, Du JL, She Q. Spironolactone Regulates HCN Protein Expression Through Micro-RNA-1 in Rats With Myocardial Infarction. J Cardiovasc Pharmacol. 2015; 65:587-92. https://doi. org/10.1097/FJC.0000000000000227.

8. Tony H, Meng K, Wu B, Yu A, Zeng Q, Yu K, Zhong Y. MicroRNA-208a Dysregulates Apoptosis Genes Expression and Promotes Cardiomyocyte Apoptosis during Ischemia and Its Silencing Improves Cardiac Function after Myocardial Infarction. Mediators Inflamm. 2015; 2015:479123. https://doi.org/10.1155/2015/479123.

9. Wang L, Ma L, Fan H, Yang Z, Li L, Wang H. MicroRNA-9 regulates cardiac fibrosis by targeting PDGFR- $\beta$ in rats. J Physiol Biochem. 2016; 72:213-223. https://doi. org/10.1007/s13105-016-0471-y.

10. Wang XW, Zhang FX, Yang F, Ding ZF, Agarwal N, Guo ZK, Mehta JL. Effects of linagliptin and liraglutide on glucose- and angiotensin II-induced collagen formation and cytoskeleton degradation in cardiac fibroblasts in vitro. Acta Pharmacol Sin. 2016; 37:1349-1358. https://doi. org/10.1038/aps.2016.72.

11. Huang W, Tian SS, Hang PZ, Sun C, Guo J, Du ZM. Combination of microRNA-21 and microRNA-146a Attenuates Cardiac Dysfunction and Apoptosis During Acute Myocardial Infarction in Mice. Mol Ther Nucleic Acids. 2016; 5:e296. https://doi.org/10.1038/mtna.2016.12.

12. Jiang X, Ning Q, Wang J. Angiotensin II induced differentially expressed microRNAs in adult rat cardiac fibroblasts. J Physiol Sci. 2013; 63:31-38. https://doi.org/10.1007/ s12576-012-0230-y.

13. Forrester HB, Temple-Smith P, Ham S, de Kretser D, Southwick G, Sprung CN. Genome-wide analysis using exon arrays demonstrates an important role for expression of extra-cellular matrix, fibrotic control and tissue remodelling genes in Dupuytren's disease. PLoS One. 2013; 8:e59056. https://doi.org/10.1371/journal.pone.0059056.

14. Li C, Han R, Kang L, Wang J, Gao Y, Li Y, He J, Tian J. Pirfenidone controls the feedback loop of the AT1R/p38 $\mathrm{MAPK} /$ renin-angiotensin system axis by regulating liver $\mathrm{X}$ receptor- $\alpha$ in myocardial infarction-induced cardiac fibrosis. Sci Rep. 2017; 7:40523. https://doi.org/10.1038/srep40523.

15. Sui X, Wei H, Wang D. Novel mechanism of cardiac protection by valsartan: synergetic roles of TGF- $\beta 1$ and HIF- $1 \alpha$ in Ang II-mediated fibrosis after myocardial infarction. J Cell Mol Med. 2015; 19:1773-1782. https://doi.org/10.1111/ jcmm.12551.
16. Thum T, Gross C, Fiedler J, Fischer T, Kissler S, Bussen M, Galuppo P, Just S, Rottbauer W, Frantz S, Castoldi M, Soutschek J, Koteliansky V, et al. MicroRNA-21 contributes to myocardial disease by stimulating MAP kinase signalling in fibroblasts. Nature. 2008; 456:980-984. https:// doi.org/10.1038/nature07511.

17. Hong Y, Cao H, Wang Q, Ye J, Sui L, Feng J, Cai X, Song H, Zhang X, Chen X. MiR-22 may Suppress Fibrogenesis by Targeting TGF $\beta$ R I in Cardiac Fibroblasts. Cell Physiol Biochem. 2016; 40:1345-1353. https://doi. org/10.1159/000453187.

18. van Rooij E, Sutherland LB, Liu N, Williams AH, McAnally J, Gerard RD, Richardson JA, Olson EN. A signature pattern of stress-responsive microRNAs that can evoke cardiac hypertrophy and heart failure. Proc Natl Acad Sci U S A. 2006; 103:18255-18260.

19. Zhang Y, Huang XR, Wei LH, Chung AC, Yu CM, Lan HY. miR-29b as a therapeutic agent for angiotensin II-induced cardiac fibrosis by targeting TGF- $\beta / \mathrm{Smad} 3$ signaling. Mol Ther. 2014; 22:974-985. https://doi.org/10.1038/ mt.2014.25.

20. Zhao X, Wang K, Hu F, Qian C, Guan H, Feng K, Zhou Y, Chen Z. MicroRNA-101 protects cardiac fibroblasts from hypoxia-induced apoptosis via inhibition of the TGF- $\beta$ signaling pathway. Int J Biochem Cell Biol. 2015; 65: 155-164. https://doi.org/10.1016/j.biocel.2015.06.005.

21. Duisters RF, Tijsen AJ, Schroen B, Leenders JJ, Lentink V, van der Made I, Herias V, van Leeuwen RE, Schellings MW, Barenbrug P, Maessen JG, Heymans S, Pinto YM, Creemers EE. miR-133 and miR-30 regulate connective tissue growth factor: implications for a role of microRNAs in myocardial matrix remodeling. Circ Res. 2009; 104:170-178. https:// doi.org/10.1161/CIRCRESAHA.108.182535.

22. Yang Z, Cappello T, Wang L. Emerging role of microRNAs in lipid metabolism. Acta Pharm Sin B. 2015; 5:145-150. https://doi.org/10.1016/j.apsb.2015.01.002.

23. Goedeke L, Salerno A, Ramírez CM, Guo L, Allen RM, Yin X, Langley SR, Esau C, Wanschel A, Fisher EA, Suárez Y, Baldán A, Mayr M, Fernández-Hernando C. Long-term therapeutic silencing of miR-33 increases circulating triglyceride levels and hepatic lipid accumulation in mice. EMBO Mol Med. 2014; 6:1133-1141. https://doi.org/10.15252/ emmm.201404046.

24. Hang P, Sun C, Guo J, Zhao J, Du Z. BDNF-mediates Down-regulation of MicroRNA-195 Inhibits Ischemic Cardiac Apoptosis in Rats. Int J Biol Sci. 2016; 12:979-89. https://doi.org/10.7150/ijbs.15071. 